\title{
TORSION OF A VECTOR FIELD
}

T. K. PAN

1. Introduction. This paper intends to generalize the concept of torsion for a vector field in a surface. Indicatric torsion is defined to include geodesic torsion as a special case. Some theorems about torsion and geodesic torsion are generalized.

Known formulas and curves which are related with this investigation are stated as follows for reference.

Let $S: x^{i}=x^{i}\left(u^{1}, u^{2}\right)$ denote a surface of class not less than 2 in three dimensional euclidean space referred to a rectangular cartesian coordinate system. ${ }^{1}$ Let $C: u^{\alpha}=u^{\alpha}(s)$ denote a curve in $S$ and of class not less than $3, s$ being its arc length. Let $v: v^{i}=x^{i},{ }_{\alpha} p^{\alpha}$ denote a unit vector field in $S$. Let $g_{\alpha \beta}$ and $d_{\alpha \beta}$ denote, respectively, the first and the second fundamental tensors of $S$ such that $g=\left|g_{\alpha \beta}\right|>0$ and $d=\left|d_{\alpha \beta}\right|$ $\neq 0$.

Let $P\left(x^{i}\right)$ denote a point of $C$ and ${ }_{v} \kappa$ the arc-rate of turning of $v$ at $P$ with respect to $C$. Then we have

$$
d v / d s={ }_{0} \kappa w
$$

where $w\left(w^{i}\right)$ is a unit vector orthogonal to $v$. The quantity ${ }_{v} \kappa$ and the vector $w$ are called, respectively, the absolute curvature and the absolute curvature vector of $v$ at $P$ with respect to $C$. The sense of $w$ is chosen in such a way as to make ${ }_{v} k>0$ so that, when $v$ coincides with the unit tangent vector $t$ of $C$ at $P$, w becomes identical with the unit normal vector $n$ of $C$ at $P$.

Denote by $\gamma$ the vector product of $v$ and $w$ in the given order, that is

$$
\gamma=v \times w .
$$

Then the formulas of Frenet for a curve in a Riemannian space $[1, \text { pp. 106-107 }]^{2}$ reduce for $v$ along $C$ in $S$ to (1.1) and

$$
\begin{aligned}
& d w / d s=-{ }_{\bullet} \kappa v-{ }_{\imath} \tau \gamma, \\
& d \gamma / d s={ }_{\imath} \tau w .
\end{aligned}
$$

Presented to the Society, December 29, 1954 under the title Angular spread and indicatric torsion; received by the editors June 28, 1955.

${ }^{1}$ All Latin indices in this paper run from 1 to 3 and all Greek indices from 1 to 2. We agree as usual that repeated indices mean summation.

2 Numbers in brackets refer to the references at the end of this paper. 
The quantity,$\tau$ may be called the torsion of $v$ at $P$ with respect to $C$.

Let $\boldsymbol{}_{\boldsymbol{\sigma}}$ and ${ }_{v} \kappa_{n}$ denote, respectively, the geodesic curvature of $v$ with respect to $C$ and the normal curvature of $v$ with respect to $C$. They are, respectively, the magnitudes of the components tangential and normal to $S$ of the absolute curvature vector of $v$ with respect to $C[\mathbf{3}]$.

The direction with respect to which ${ }_{v} \kappa_{n}$ at $P$ has its extreme value is the principal direction of $v$ at $P$ and the corresponding extreme value is the principal curvature of $v$ at $P$. A curve in $S$, whose direction at each and every point is a principal direction of $v$, is a line of curvature of $v$. It is defined by

$$
\epsilon^{\alpha \beta} g_{\alpha \gamma} d_{\beta \delta} p^{\delta} d u^{\gamma}=0 .
$$

The unit vectors in the principal directions of $v$ in $S$ form a unit vector field called the unit principal vector field of $v$ and those in a family of principal directions of $S$ form a unit principal vector field of $S$. Evidently the unit principal vector field of a unit principal vector field of $S$ is identical with that unit principal vector field of $S$.

A direction with respect to which ${ }_{\nu} \kappa_{n}$ is zero is called an asymptotic direction of $v$. A curve in $S$, whose direction at each and every point is an asymptotic direction of $v$, is an asymptotic line of $v$. It is defined by

$$
d_{\delta \gamma} p^{\delta} d u^{\gamma}=0 .
$$

The unit vectors in the asymptotic directions of $v$ in $S$ form a unit vector field called the unit asymptotic vector field of $v$ and those in a family of asymptotic directions of $S$ form a unit asymptotic vector field of $S$. Evidently the unit asymptotic vector field of a unit asymptotic vector field of $S$ coincides with that unit asymptotic vector field of $S$.

A curve of a unit vector field $v$ in $S$ is a curve in $S$, along which the vectors of $v$ are tangent to the curve. It is defined by

$$
\frac{d u^{\alpha}}{d s}=p^{\alpha} \text {. }
$$

It is obvious that the curve and the line of curvature of a unit principal vector field of $S$ at $P$ are identical with the corresponding line of curvature of $S$ at $P$. Similarly, the curve and the asymptotic line of a unit asymptotic vector field of $S$ coincide with the corresponding asymptotic line of $S$ at $P$.

It is shown that the asymptotic lines of $v$ form a conjugate net with the curves of $v$ in $S$ and form an orthogonal net with the lines of curvature of $v$ in $S$. 
The three quantities ${ }_{\nu} \kappa_{{ }} \kappa_{g}$, and ${ }_{\bullet} \kappa_{n}$ are found to possess the following relation

$$
\frac{d v^{i}}{d s}={ }_{\diamond} \kappa w^{i}={ }_{{ }} \kappa_{0} q^{\alpha} x^{i}, \alpha+{ }_{{ }} \kappa_{n} X^{i}
$$

where

$$
{ }_{\bullet} \kappa_{\theta} q^{\alpha}=p^{\alpha}, \gamma \frac{d u^{\gamma}}{d s}, \quad{ }_{\bullet} \kappa_{n}=d_{\delta \gamma} p^{\delta} \frac{d u^{\gamma}}{d s}
$$

and where $X^{i}$ are the components of the unit vector $X$ normal to $S$ at $P$.

2. Generalized theorems of Enneper and Bonnet. Let $\omega$ be the angle between $X$ and $w$. From (1.2) we have

$$
\sin \omega=\boldsymbol{\gamma} \cdot X \text {. }
$$

Differentiating (2.1) with respect to $s$ and simplifying by (1.3), we obtain

$$
\left(\frac{d \omega}{d s}-{ }_{\bullet} \tau\right) \cos \omega=\gamma \cdot \frac{d X}{d s} .
$$

Since $\gamma$ is linearly dependent on $X$ and $\mu: \mu^{i}=q^{\alpha} x^{i},{ }_{\alpha}$, it can be readily found that

$$
\gamma=X \sin \omega-\mu \cos \omega .
$$

Substitution of (2.3) into (2.2) gives

$$
\left(\frac{d \omega}{d s}-, \tau\right) \cos \omega=-\mu \cdot \frac{d X}{d s} \cos \omega .
$$

Note that

and that

$$
\frac{d X^{i}}{d s}=-d_{\alpha \gamma} g^{\gamma \beta} x^{i},{ }_{\beta} \frac{d u^{\alpha}}{d s}
$$

$$
\mu^{i}=e \epsilon^{\sigma \alpha} g_{\sigma \beta} p^{\beta} x^{i}, \alpha
$$

where $e$ is plus or minus one so that $\mu$ makes a right angle with $v$ $[2$, p. 132 and p. 187]. Hence equation (2.4) is equivalent to

$$
\left(\frac{d \omega}{d s}-\imath \tau+\imath_{\imath}\right) \cos \omega=0
$$

where 


$$
{ }_{{ }^{\circ} \tau_{0}}=e e^{\beta \alpha} g_{\alpha \delta} d_{\gamma \beta} p^{\delta} \frac{d u^{\gamma}}{d s} .
$$

When $\cos \omega \neq 0$, that is, when $C$ is not an asymptotic line of $v$, we have from (2.5)

$$
{ }_{\bullet} \tau=\frac{d \omega}{d s}+{ }_{{ }} \tau_{g} .
$$

It can be shown that equation (2.7) holds for $\cos \omega=0$ as follows.

When $\cos \omega=0, \omega=\pi / 2$ and $C$ is an asymptotic line of $v$. From (1.5) we find its unit tangent vector with a choice of positive direction is given by

$$
\frac{d u^{\gamma}}{d s}=\frac{\epsilon^{\rho \gamma} d_{\delta \rho} p^{\delta}}{\left(h_{\lambda \tau} p^{\lambda} p^{\gamma}\right)^{1 / 2}}
$$

where $h_{\alpha \beta}=\sum_{i}\left(\partial X^{i} / \partial u^{\alpha} \cdot \partial X^{i} / \partial u^{\beta}\right)$. In this case, $w^{i}=e q^{\alpha} x^{i}{ }_{, \alpha}$ as follows from (1.7) and $\gamma=e X$ as follows from (1.2), $e$ being +1 or -1 as the case may be. Consequently we have from (1.3)

$$
{ }_{{ } \tau} w^{i}=-\frac{e d_{\alpha \gamma} g^{\gamma \beta} x^{i},{ }_{\beta} \epsilon^{\rho \alpha} d_{\delta \rho} p^{\delta}}{\left(h_{\lambda \tau} p^{\lambda} p^{\tau}\right)^{1 / 2}}
$$

which yields

$$
{ } \tau=-\frac{e d}{g\left(h_{\lambda \tau} p^{\lambda} p^{\tau}\right)^{1 / 2}} .
$$

Substituting (2.8) for $d u^{\gamma} / d s$ in (2.6), we obtain ${ }_{v} \tau_{g}={ }_{v} \tau$. Thus (2.7) holds also for $\cos \omega=0$, since $\omega$ is constant in this case.

It is found [3, pp. 959-960] that the square of the principal curvature of the unit vector field $v$ in $S$ at $P$ is equal to

$$
\left({ }_{v} \bar{\kappa}_{n}\right)^{2}=h_{\alpha \beta} p^{\alpha} p^{\beta}=M \kappa_{n}-K
$$

where $M$ and $K$ are, respectively, the mean curvature and the Gaussian curvature of $S$ at $P$ and where $\kappa_{n}$ is the normal curvature of the curve of $v$ at $P$. It is well known that the Gaussian curvature at a point of $S$ is equal to $d / g$ at the point [2, p. 225]. Hence from (2.9) we have

THEOREM 2.1. The torsion of a unit vector field in a surface at a point with respect to the asymptotic line of the field at the point is numerically equal to the ratio of the Gaussian curvature of the surface at the point and the principal curvature of the field at the point.

When the unit vector field $v$ is a unit asymptotic vector field of 
$S$, the asymptotic line of $v$ at $P$ has its normal curvature equal to zero. Hence Theorem 2.1 reduces, in consequence of $(2.10)$, to the theorem of Enneper [2, p. 248], of which Theorem 2.1 is an appropriate generalization.

Since the lines of curvature of $S$ form an orthogonal net in $S$, the family of asymptotic lines of a unit principal vector field of $S$ which corresponds to one family of lines of curvatures of $S$ is identical with the other family of lines of curvature of $S$. Hence, by (2.10), Theorem 2.1 yields a relation between the principal normal curvature of $S$ at $P$ and the torsions of each unit principal vector field of $S$ with respect to the curve of the other unit principal vector field of $S$ at $P$.

THEOREM 2.2. The torsion of a unit vector field corresponding to one family of lines of curvature of a surface with respect to the line of curvature of the second family of the surface at a point is numerically equal to the normal curvature in the direction of the line of curvature of the second family at the point.

Since the quantity ${ }_{v} \tau_{0}$ for a given vector field is a function of a point and a direction at a point as follows from (2.6), the quantity ${ }_{v} \tau-d \omega / d s$, which is equal to $\tau_{0}$ from (2.7), is the same for a given vector field with respect to all curves at a point having a common tangent. It is equal to ${ }_{v} \tau$ with respect to any curve for which $\omega$ is constant. Accordingly ${ }_{v} \tau_{0}$ is called the indicatric torsion of a vector field in a direction, which includes the geodesic torsion of a curve as a special case.

Relation (2.7) is similar to a relation between ordinary torsion and geodesic torsion due to Bonnet. It comprises the following theorem.

TheOREM 2.3. If $v$ is a unit vector field in a surface $S$ and $C$ is a curve in $S$ through the point $P$ in a given direction, the difference, at $P$, of the torsion of $v$ with respect to $C$ and the rate of change, with respect to the arc of $C$, of the angle which the plane determined by $v$ and its absolute curvature vector at $P$ along $C$ makes with the tangent plane to $S$ is the same for $v$ with respect to all curves which pass through $P$ and have at $P$ the given direction, and is equal to the indicatric torsion of $v$ at $P$ in the given direction.

3. Indicatric torsion. Let $C_{0}$ be the asymptotic line of a unit vector field $v$ at $P$ and $C_{1}, C_{2}$ two curves making equal angles with $C_{0}$ at $P$. Denote the unit tangent vectors at $P$ of $C_{0}, C_{1}$ and $C_{2}$ by $l\left(l^{\alpha}\right), m\left(m^{\alpha}\right)$ and $n\left(n^{\alpha}\right)$, respectively. Then from (2.8) we have

$$
l^{\gamma}=\epsilon^{\rho \gamma} d_{\delta_{\rho}} p^{\delta} /\left(h_{\lambda \tau} p^{\lambda} p^{\tau}\right)^{1 / 2} .
$$

Substitution of (3.1) into 


$$
g_{\alpha \beta} l^{\alpha} m^{\beta}=g_{\gamma \delta} l^{\gamma} n^{\delta}
$$

gives

$$
\epsilon^{\rho \alpha} g_{\alpha \beta} d_{\delta \rho} p^{\delta} m^{\beta}=\epsilon^{\mu \gamma} g_{\gamma \nu} d_{\lambda \mu} p^{\lambda} n^{\nu}
$$

which yields by (2.6) the following theorem.

THEOREM 3.1. The indicatric torsions of a unit vector field in a surface at a point in two directions which are equally inclined to the asymptotic direction of the field are equal.

The unit asymptotic vector field of $v$ is defined by (2.8). Substituting (2.8) for $\rho^{\delta}$ and (1.6) for $d u^{\gamma} / d s$ in (2.6) we obtain

$$
{ }_{\bullet} \tau_{0}=\frac{e \epsilon^{\beta \alpha} g_{\alpha \delta} d_{\gamma \beta} \epsilon^{\rho \delta} d_{\sigma \rho} p^{\sigma} p^{\gamma}}{\left(h_{\lambda \tau} p^{\lambda} p^{\tau}\right)^{1 / 2}}=e\left(h_{\alpha \beta} p^{\alpha} p^{\beta}\right)^{1 / 2}
$$

which yields

THEOREM 3.2. The indicatric torsion of the unit asymptotic vector field of a unit vector field in a surface at a point with respect to the curve of the latter is numerically equal to the principal curvature of the latter at the point.

From (1.4) and (2.6) we have

THEOREM 3.3. The indicatric torsion of a unit vector field in a surface at a point with respect to a curve is zero if and only if the curve is the line of curvature of the field at the point.

When the vector field is a unit principal vector field of $S$, the line of curvature of the field at $P$ is the corresponding line of curvature of $S$ at $P$. Hence Theorem 3.3 is a generalization of a theorem [2, p. 248] which may read as follows:

THEOREM 3.4. A necessary and sufficient condition that the indicatric torsion of a unit vector field in a surface at a point with respect to the curve of the field at the point be zero is that the vector field be a unit principal vector field of the surface.

The above theorem can be obtained also directly from (2.6) by substituting $p^{\gamma}$ for $d u^{\gamma} / d s$, since a vector field $v$ in $S$ is a principal field of $S$ if and only if

$$
\epsilon^{\alpha \beta} d_{\alpha \gamma} g_{\beta \delta} p^{\gamma} p^{\delta}=0 .
$$

Solve (1.4) for $d u^{\gamma} / d s$ which, with a choice of positive direction, is given by 


$$
\frac{d u^{\gamma}}{d s}=\frac{g^{\gamma \beta} d_{\beta \delta} p^{\delta}}{\left(h_{\lambda \tau} p^{\lambda} p^{\tau}\right)^{1 / 2}} .
$$

The unit vectors defined by (3.4) form the unit principal vector field of $v$. Substituting (3.4) for $v$ in (2.6) where $d u^{\gamma} / d s$ is replaced by (2.8) we obtain

$$
{ }_{{ }^{\prime} \tau_{o}}=-\frac{e K d_{\alpha \beta} p^{\alpha} p^{\beta}}{h_{\lambda \tau} p^{\lambda} p^{\tau}}
$$

which is equal to zero if and only if $v$ is in an asymptotic direction of $S$ at $P$. By Ex. $16[4$, p. 182], equation (3.5) may be written as

$$
{ }_{\bullet} \tau_{\theta}=\frac{e K \cos \theta}{d s \cdot d \bar{s}}
$$

where $\theta$ is the angle between $v$ and its spherical image and where $d s$ and $d \bar{s}$ are respectively the linear element of the curve of $v$ and the linear element of its spherical image. Hence we have

TheOREM 3.5. The indicatric torsion of the unit principal vector field of a given unit vector field in a surface at a point with respect to the asymptotic line of the given field at the point is numerically equal to the ratio of two products: the first is the product of the Gaussian curvature of the surface and cosine of the angle between the curve of the given field and its spherical image at the point; the second is the product of the linear element of the curve of the given field and the linear element of its spherical image at the point. It is identically zero if and only if the given field is a unit asymptotic vector field of the surface.

When we substitute (3.4) for $p^{\delta}$ in (2.6) where $d u^{\gamma} / d s$ is replaced by $p^{\gamma}$, we obtain the following result

$$
{ }_{v} \tau_{g}=e K \epsilon_{\alpha \beta} p^{\alpha} p^{\beta} /\left(h_{\lambda \tau} p^{\lambda} p^{\tau}\right)^{1 / 2}
$$

which is always equal to zero. Hence we have

THEOREM 3.6. The indicatric torsion of the unit principal vector field of a given unit vector field in a surface with respect to the curve of the given field is always equal to zero.

When (3.4) is substituted for both $p^{\delta}$ and $d u^{\gamma} / d s$ in (2.6), we obtain with the aid of $(2.10)$ and $[2$, p. 247]

$$
{ }_{v} \tau_{g}=\frac{e K}{\left({ }_{v} \bar{K}_{n}\right)^{2}} \tau_{g}
$$


where $\tau_{0}$ is the geodesic torsion of the curve of the vector field $v$. Hence we have

THEOREM 3.7. The indicatric torsion of the unit principal vector field of a given unit vector field in a surface at a point with respect to the curve of that unit principal vector field is proportional to the geodesic torsion of the curve of the given field at the point, the factor of proportionality being numerically the quotient of the Gaussian curvature of the surface and the square of the principal curvature of the given field at the point.

4. Torsion of a surface. We shall find the direction in $S$ at $P$ in which the indicatric torsion of $v$ in $S$ at $P$ attains its extreme value. Such direction is known as the principal direction of the torsion of $v$ at $P$ and the corresponding indicatric torsion is called the principal torsion of $v$ at $P$. A curve on $S$, whose direction at each and every point is a principal direction of the torsion of $v$ is called a line of torsion of $v$. The lines of torsion of $v$ form a family of curves in $S$.

The principal direction of the torsion of $v$ and the line of torsion of $v$ are found from (2.6) by setting $\partial\left({ }_{v} \tau_{g}\right) / \partial\left(d u^{\alpha}\right)$ equal to zero. The resulting equation after simplification is

$$
g_{\alpha \beta} g_{\gamma \delta} d^{\alpha \gamma} p^{\beta} d u^{\delta}=0 .
$$

The family of orthogonal trajectories of the lines of torsion of $v$ in $S$ is defined by

$$
g_{\sigma \gamma} \epsilon^{\beta \sigma} g_{\alpha \delta} g_{\beta \rho} d^{\alpha \rho} p^{\delta} d u^{\gamma}=0
$$

which can be shown readily to be equivalent to (1.4). Since the asymptotic lines of $v$ form an orthogonal net with the lines of curvature of $v$ in $S$, we conclude that (4.1) is identical with (1.5). Hence by Theorem 2.1 we have

THEOREM 4.1. The principal direction of the torsion of a unit vector field in a surface at a point coincides with the asymptotic direction of the field at the point, which is conjugate to the vector of the field at the point and orthogonal to the principal direction of the field at the point. The principal torsion of the field at the point is numerically equal to the ratio of the Gaussian curvature of the surface at the point and the principal curvature of the field at the point. The family of lines of torsion of the field is identical with the family of asymptotic lines of the field.

Noting that $K=d / g$ and $g_{\alpha \beta} p^{\alpha} p^{\beta}=1$, we may write equation (2.9) in the following form

$$
{ }_{v} \tau=-e K\left(\frac{g_{\alpha \beta} p^{\alpha} p^{\beta}}{h_{\lambda \tau} p^{\lambda} p^{\tau}}\right)^{1 / 2}
$$


The extreme values of the principal torsion of $v$ at $P$ as $v$ varies are given by those unit vector fields $v$ for which $\partial\left({ }_{v} \tau\right) / \partial p^{\delta}=0$ in (4.2), that is

$$
\epsilon^{\alpha \beta} h_{\alpha \gamma} g_{\beta \delta} p^{\gamma} p^{\delta}=0 .
$$

Thus there are two unit vector fields in $S$ which have the extreme values of the extreme values (4.2) at $P$. We call these two extreme values the principal torsions of $S$ at $P$. Their product and sum are called the total torsion of $S$ at $P$ and the mean torsion of $S$ at $P$, respectively.

Since $h_{\alpha \beta}=d_{\alpha \beta} M-g_{\alpha \beta} K[2$, p. 253], equation (4.3) is reducible to (3.3) which defines the lines of curvature of $S$. Thus the two principal unit vector fields of $S$ not only offer the extremal principal curvatures of all vector fields in $S$ at $P$, but they give as well the extremal principal torsions of all vector fields in $S$ at $P$. From Theorem 2.2 the total torsion of $S$ at $P$ and the mean torsion of $S$ at $P$ are respectively equal to the Gaussian curvature of $S$ at $P$ and the mean curvature of $S$ at $P$. The content of the "theorema egregium" naturally holds true for the total torsion of $S$ at $P$. Hence we have

TheOREM 4.2. The total torsion of a surface is a bending invariant. The total and mean torsions of a surface at a point are respectively equal to the total and mean curvatures of the surface at the point. The two unit principal vector fields of a surface are the unit vector fields in the surface which have the extremal principal torsions of all vector fields in the surface at a point.

Weatherburn called the Gaussian or total curvature of a surface as the second curvature of the surface because it is a differential invariant of higher order, similar in many respects to the torsion or second curvature of a curve [4, pp. 264-265]. His terminology is also justified in a certain sense by the above theorem.

\section{REFERENCES}

1. L. P. Eisenhart, Riemannian geometry, Princeton University Press, 1949.

2. - An introduction to differential geometry with the use of the tensor calculus, Princeton University Press, 1947.

3. T. K. Pan, Normal curvature of a vector field, Amer. J. Math. vol. 75 (1952) pp. $955-966$.

4. C. E. Weatherburn, Differential, geometry of three dimensions, Cambridge University Press, 1927.

UNIVERSITY OF OKLAHOMA 